Monatsschr Kinderheilkd 2021 • 169:465-466 https://doi.org/10.1007/s00112-021-01157-1 Angenommen: 2. März 2021

Online publiziert: 25. März 2021

(C) Springer Medizin Verlag GmbH, ein Teil von Springer Nature 2021

\section{Redaktion}

U. Gaiser, Maulbronn

\section{Franziska Krampe ${ }^{1}$ Ulrike Gaiser ${ }^{2}$}

${ }^{1}$ Kinderkliniken München Schwabing und Harlaching und Klinikum Rechts der Isar, Technische Universität München, München, Deutschland

${ }^{2}$ Klinik für Kinderneurologie und Sozialpädiatrie, Kinderzentrum Maulbronn, Maulbronn, Deutschland

\title{
Forschung und Klinik - Clinical Scientist
}

Das Studium ist fertig, die Doktorarbeit auch. Während der ersten Berufsjahre stellt man dann fest, dass man zwar einerseits die klinische Arbeit mit den kleinen Patienten schätzt, aber andererseits auch gern im Labor steht oder klinische Studien durchführt. Viele wünschen sich daher eine gute Vereinbarkeit von klinischer Tätigkeit und Forschungsmöglichkeiten, um einen $\mathrm{PhD}$ oder eine Habilitation zu erwerben, oder einfach, weil man Lust an Forschung hat. Zumeist ist aber nicht beides mit vollem Einsatz möglich; die jungen MedizinerInnen sind häufig zwischen ihren Pflichten hin- und hergerissen und als „Diener zweier Herren“ im Doppeljob schnell ausgebrannt. Zu oft hat das „Vereinbaren“ bedeutet, dass man zusätzlich zu $100 \%$ klinischer Tätigkeit abends noch Zeit in die Forschung investierte. Es geht aber auch anders: Es gibt nun deutschlandweit zunehmend strukturierte Programme, die eine gute Vereinbarkeit von Forschung und klinischer Tätigkeit ermöglichen sollen: ClinicalScientist-Programme. Diese können sowohl durch die (Uni)kliniken direkt vollständig finanziert sein als auch im Rahmen von Stipendien z. B. von der Deutschen Forschungsgemeinschaft oder dem Bundesministerium für Bildung und Forschung finanziell unterstützt sein.

\section{》) Clinical-Scientist-Programme sollen die Vereinbarkeit von Forschung und klinischer Tätigkeit fördern}

Frau Prof. Ingeborg Krägeloh-Mann, Universitätsprofessorin a. D., Vizepräsi- dentin der Deutschen Gesellschaft für Kinder- und Jugendmedizin (DGKJ), Ombudsfrau der Medizinischen Fakultät, Abteilung Neuropädiatrie, Entwicklungsneurologie, Sozialpädiatrie am Universitätsklinikum Tübingen und langjährige Chefärztin erzählt von verschiedenen Angeboten für junge forschungsinteressierte Kolleg*innen:

An unserer Fakultät gibt es mehrere Graduiertenprogramme und -akademien, meist interdisziplinär, sowie Formate für junge Wissenschaftler in unterschiedlichen Phasen ihrer Karriere - nach der Promotion, kurz vor der Habilitation und danach - auch Programme spezifisch an Frauen gerichtet, oder Programme zur Vorbereitung des ersten DFG-Antrags, für Pilotprojekte, um klinische Studien beantragen zu können, Antragsformate als Clinical Scientist oder für eine „Junior Research Group", oder um ein Jahr in einem anderen Institut eine Methodik zu erlernen - dies wird in der sog. Junior Academy zusammengefasst, einer Plattform, die Organisations- und Strukturhilfe für eine akademische Karriere geben will. Eine Vereinbarkeit von Forschung und Klinik, von Familie und Beruf ist hier ein wichtiges Thema. Die Medizinische Fakultät gibt dafür mehr als 6 Mio. $€$ pro Jahr aus. An unserer Klinik speziell gibt es ein sog. Forschungs-Retreat jedes Jahr, zu dem insbesondere Nachwuchswissenschaftler eingeladen sind, auch schon Studenten während der Promotion, damit man seine Projekte vorstellen kann, andere Arbeitsgruppen kennenlernen kann. Und im Prinzip hat jede wissenschaftliche Arbeitsgruppe ihren Austausch in Treffen.
Zudem gibt es ihrer Meinung nach in den letzten Jahren positive Entwicklungen, um die Vereinbarkeit zwischen Forschung Klinik gleichermaßen zu ermöglichen:

Formal haben sich m.E. die Bedingungen für eine Vereinbarkeit von Forschung und Klinik in den letzten Jahren/wenigen Jahrzehnten verbessert, da es allen medizinischen Fakultäten klar ist, dass sie dafür Angebote machen müssen, da Forschung im Alltag neben der Klinik kaum umsetzbar ist. Die Klinikchefs an Universitätsklinika haben als Lehrstuhlinhaber einen Auftrag für Forschung und werden an ihrer wissenschaftlichen Leistung gemessen, haben daher hohes Interesse, junge ärztliche Mitarbeiter zu gewinnen, die neben einem Interesse für die klinische Weiterbildung auch Forschung machen wollen. Clinical-Scientist-Programme zielen explizit darauf ab, klinische Tätigkeit mit Forschung zu verbinden. Aber auch sonst werden in Forschungsprojekten oft Ärzte in Teilzeit eingeplant/-gestellt, damit sie auch weiter klinisch tätig sein können. Forschungstätigkeit wird auch im Rahmen der Weiterbildung anerkannt, insbesondere wenn sie einen klinischen Bezug hat - üblicherweise sind dies 6 Monate, darüber hinaus ist mit den jeweiligen Ärztekammern auszuhandeln. Trotzdem muss man mit den jungen Assistent*innen oft vereinbaren, dass ihre Weiterbildungszeit sich durch eine Forschungstätigkeit verlängert - also es braucht auch von deren Seite einen Beitrag zu den Bedingungen. Dies gilt insbesondere, wenn man eine Forschungstätigkeit für eine gewisse Zeit zu $100 \%$ einplant - was aber durchaus ein empfehlenswertes Konzept für eine 
akademische Laufbahn ist, insbesondere zu deren Beginn (s. unten Forschungsstipendien). Hat man einen „wissenschaftlichen Grundstein" gelegt, kann man dann im Verlauf leichter Klinik und Forschung kombinieren.

Eine Herausforderung ist es, die Forschungszeit der Klinikzeit gegenüber zu verteidigen - für beide Seiten - Chef und Assistent. Assistent ${ }^{*}$ in: Man will helfen, wenn jemand ausfällt, man liebt ja die klinische Tätigkeit; die Forschungsarbeit meldet keine solche Dringlichkeit an aber man weiß auch, dass die Zeit schnell vergeht und man sie braucht, für die Erreichung des Ziels im wissenschaftlichen Projekt. Chef*in: Es darf keine Assistenten erster und zweiter Qualität geben, klinische und wissenschaftliche Tätigkeit sind beide wertvoll und müssen ihren Platz erhalten.

Einen Unterschied der Bedingungen für klinische und Grundlagenwissenschaft gibt es m. E. nicht.

Eine Voraussetzung dafür, dass ein junger Kollege/eine junge Kollegin erfolgreich Forschung und Klinik bewältigen kann, sieht Frau Prof. Krägeloh-Mann in einer guten Promotionsarbeit mit Veröffentlichung, die üblicherweise für Anträge nach der Promotion eine Voraussetzung darstellt, aber nicht unbedingt im gleichen Feld geschrieben sein muss, für das der aktuelle Antrag gestellt wird.

\section{》) Assistent*innen sollten \\ sich auf eine längere \\ Weiterbildungszeit einlassen}

Zudem empfiehlt sie das Coaching eines AG-Leiters, da Mitglied in einer Arbeitsgruppe zu sein/zu werden, ein wichtiger Startpunkt für eine Forschungslaufbahn ist. Auch sollte man sich als Assistent ${ }^{*}$ in auf eine längere Weiterbildungszeit, ggf. für einen Auslandsaufenthalt (sehr zu empfehlen; Forschungsstipendienanträge dafür haben bei der DFG eine $75 \%$ ige Förderchance!) einlassen. Des Weiteren rät sie jungen Kolleg*innen, die gern in beiden Bereichen tätig sein wollen, sich einen/eine Mentor/in zu suchen (der/die eigene Chef/Chefin oder dessen/deren
Empfehlung) sowie die eigenen Wünsche sehr offen zu besprechen.

H. G. (27), Assistenzarzt der Pädiatrie an einem Universitätsklinikum in München und zum Erwerb eines $\mathrm{PhD}$ in der Forschung tätig, erzählt von seinen Erfahrungen:

Warum hast Du Dich für ein Clinical-Scientist-Programm entschieden?

Das Faszinierende an der Medizin ist es, Patientenversorgung und Forschung zu verbinden. Ein PhD zusätzlich zur Facharztausbildung ermöglicht es, die wissenschaftlichen Arbeitsweisen zu erlernen, eigene Ideen zu entwickeln und (internationale) Kontakte in Forschung und Klinik zu knüpfen.

Wie sieht Dein Arbeitsalltag aus? Was läuft gut, was sind Herausforderungen?

Während der ersten 1,5 Jahre des PhD kamen zur Vollzeitarbeit im Labor Nachtdienste und Wochenenddienste in der Klinik dazu. Die gedankliche Umstellung von einem Experiment tagsüber zum Nachtdienst war anstrengend, insbesondere ohne klinische Routine.

\section{Was hilft Dir bei der Vereinbarkeit von Forschung und Klinik?}

Unterstützung ist wichtig: Für die Klinik sind Kollegen hilfreich, die einem Praktisches beibringen, „standard operating procedures" (SOP) und Literatur zeigen oder von ihrer eigenen klinischen Erfahrung berichten. In der Forschung bringen einen der Austausch, Diskussion und Fragen weiter. Experimente brauchen einen Plan, gute Vorbereitung, Ruhe bei der Durchführung und Aufmerksamkeit bei der Auswertung. Indem man dann phasenweise den Fokus ganz auf die Forschung legt, kommt man besser voran. Die zweite Hälfte des PhD ohne klinische Tätigkeit, in der ich mich aktuell befinde, ist eine gute Entscheidung, welche wiederum durch (Drittmittel-)Förderung und ein kollegiales Team ermöglicht wird.

Hast Du Tipps für junge Kollegen, was bei einem solchen Weg hilfreich ist?

Zwischendurch hilft es, einen Schritt zur Seite zu machen oder Gespräche mit Freunden, Familie oder Kollegen zu füh- ren, um eine andere Perspektive $z u$ bekommen. Nahezu jeder PhD-Student kann bestätigen, dass es in den ersten 2 Jahren Geduld und Nerven braucht, bis man eingelesen ist, die verwendeten $\mathrm{Me}$ thoden im Labor beherrscht und besser versteht, was man tut.

\section{Wie sehen Deine Perspektiven aus? Wie soll es weitergehen?}

Nach dem PhD freue ich mich auf eine Vervollständigung meiner Facharztausbildung, möchte die Forschung aber unbedingt fortführen. Ideen, Fragen und Experimente in innovative Therapien für unsere Patienten umzusetzen, ist etwas Besonderes, woran ich auf jeden Fall weiterarbeiten will.

Dem Thema Vereinbarkeit von Forschung und Beruf widmet sich auch die Kommission für Hochschulfragen der DGKJ, die einen Workshop mit dem Titel „Karrierewege in der Wissenschaft (Clinical and Medical Scientist)“ plant. Weitere Informationen dazu findet Ihr auf der Homepage der DGKJ.

\section{Korrespondenzadresse}

\section{Franziska Krampe}

Kinderkliniken München Schwabing und Harlaching und Klinikum Rechts der Isar, Technische Universität München Ismaninger Straße 22, 81675 München, Deutschland

franziska.krampe@mri.tum.de

Interessenkonflikt. F. Krampe und U. Gaiser geben an, dass kein Interessenkonflikt besteht. 